\title{
О двух предполных классах трехзначной логики Лукасевича
}

\author{
Н. Н. ПРЕЛОВСКИЙ
}

\begin{abstract}
Two submaximal classes of 3-valued functionally complete iterative system are characterized in the paper. These two classes are functionally precomplete classes of the famous Łukasiewicz's logic.

Keywords: Łukasiewicz logic, functional class, submaximal class, iterative system
\end{abstract}

Данная работа посвящена анализу вопросов, связанных с критерием функциональной полноты замкнутых классов функций, соответствующих различным трехзначным логикам. Известный критерий функциональной полноты был сформулирован А.В. Кузнецовым и приводится в [11]. Необходимым условием для применения данного критерия является перечисление всех предполных классов, содержащихся в исследуемом классе. Поиск предполных классов систем функций, соответствующих различным трехзначным логикам, приобретает в связи с этим немаловажное значение в решении вопроса о функциональной полноте.

В работе С.В. Яблонского [12] содержится описание восемнадцати предполных классов трехзначной логики Поста $\mathrm{P}_{3}$, что позволяет сформулировать необходимое и достаточное условие полноты в этой системе. М.Ф. Раца в [7] описал десять предполных классов трехзначной логики Гейтинга $\mathrm{H}_{3} S_{0}, \ldots, S_{9}$. B.К. Финн в [9] дал описание одиннадцати предполных классов трехзначной логики Бочвара $\mathrm{B}_{3}$. Работы Раца и Финна также позволяют сформулировать необходимые и достаточные условия полноты замкнутого класса функций в $\mathrm{H}_{3}$ и $\mathrm{B}_{3}$.

Однако для трехзначной логики Лукасевича $L_{3}$, являющейся, как $\mathrm{H}_{3}$ и $\mathrm{B}_{3}$, функционально неполной (т. е. $L_{3} \subset \mathrm{P}_{3}$ и $\mathrm{P}_{3} \not \subset L_{3}$ ), 
аналогичные результаты отсутствуют. Из теоремы 2 в [7] следует, что $\mathrm{H}_{3}$ предполна в $L_{3}$. Тем не менее, в известной литературе отсутствует описание предполных в $L_{3}$ классов функций, отличных от $\mathrm{H}_{3}$. Ниже будут рассмотрены два других предполных класса трехзначной логики Лукасевича, а также приведены доказательства их предполноты.

\section{1 Основные понятия}

Сделаем предварительно несколько замечаний, касающихся используемой нотации, а также дадим определения основных понятий, встречающихся в настоящей работе.

В качестве переменных для аргументов функций используются латинские буквы $x, y, z$, возможно с индексами. Для обозначения значений переменных используются греческие буквы $\alpha$, $\beta$ и $\gamma$ также возможно с индексами. Запись функций осуществляется с использованием известного понятия формулы логики высказываний [7]. Аргументы функций, как и сами функции, принимают значения из множества $\{1,1 / 2,0\}$. Значение $1 / 2$ будем называть промежуточным. В рассмотрении используются понятия операции суперпозиции, замыкания системы функций относительно операции суперпозиции, замкнутого класса, функционально полного и предполного классов, а также понятие базиса.

Дадим вначале определение операции суперпозиции.

ОПРЕДЕЛЕНИЕ 1. Если имеется система функций

$$
\left\{f_{1}\left(x_{1}, \ldots, x_{n}\right), \ldots, f_{r}\left(x_{1}, \ldots, x_{n}\right)\right\},
$$

то суперпозицией функций данной системы называется либо функция, полученная из уже имеющихся функций путем замены переменных, либо, если установлено, что функции $f_{j}^{i}\left(x_{1_{i j}}, \ldots\right.$, $\left.x_{n_{i j}}\right), \ldots, f_{m}^{k}\left(x_{1_{k m}}, \ldots, x_{n_{k m}}\right)$, а также функция $f_{t}^{s}\left(x_{j_{s t}}, \ldots, x_{m_{s t}}\right)$ являются суперпозициями исходной системы, то и функция

$$
f_{t}^{s}\left(f_{j}^{i}\left(x_{1_{i j}}, \ldots, x_{n_{i j}}\right), \ldots, f_{m}^{k}\left(x_{1_{k m}}, \ldots, x_{n_{k m}}\right)\right)
$$

также является суперпозицией функций данной системы.

Говоря неформально, речь идет о всевозможных подстановках вместо аргументов исходной системы функций. Буквы $F$ и $G$ 
будем в дальнейшем использовать для обозначения произвольных систем и классов функций.

В [4], [11], [12] содержится определение замыкания и замкнутого класса функций.

ОПРЕДЕЛЕНИЕ 2. Множество $[F]$ называется замыканием системы (класса) функций $F$, если оно содержит все суперпозиции функций над классом $F$ и не содержит никаких других функций.

Оператор замыкания [ . . ] удовлетворяет следующим четырем условиям:

- $F \subseteq[F]$

- $[[F]]=[F]$

- $F \subseteq G \Rightarrow[F] \subseteq[G]$

- Множество функций $\mathrm{F}$ замкнуто, если $F=[F]$

ОПРЕДЕЛЕНИЕ 3. Систему функций называем базисом данного класса функций, если она эквивалентна этому классу, но никакая ее собственная подсистема не эквивалентна ему. Система функций $G$, эквивалентная классу $F$, называется (функционально) полной в этом классе, т. е. G (функционально) полна в $F \Leftrightarrow$ def $[G]=F$

ОПРЕДЕЛЕНИЕ 4. Система функций $\mathrm{G}$, функционально полная в классе $F=P_{k}$, где $\mathrm{P}_{k}$ есть $k$-значная логика Поста, называется функционально полной (о логиках Поста см. [5, с. 88-91]).

Самым важным для дальнейшего рассмотрения является следующее понятие предполного в F класса.

ОПРЕДЕЛЕНИЕ 5. Если $G$ и $F$ - замкнутые классы функций и $G \subset F$, но $F \not \subset G$, то $G$ называется предполным в $F$, если и только если замыкание объединения класса $G$ и функции $f\left(x_{1}, \ldots, x_{n}\right)$ такой, что $f\left(x_{1}, \ldots, x_{n}\right) \in F$ и $f\left(x_{1}, \ldots, x_{n}\right) \notin$ $G$, совпадает с $F$, т. е. $G$ предполон в $F \Leftrightarrow_{d e f} f\left(x_{1}, \ldots, x_{n}\right) \in$ $F \& f\left(x_{1}, \ldots, x_{n}\right) \notin G \Rightarrow\left[G \cup f\left(x_{1}, \ldots, x_{n}\right)\right]=F$, где $\left[G \cup f\left(x_{1}, \ldots\right.\right.$, $\left.x_{n}\right)$ ] есть замыкание теоретико-множественного объединения.

ЗАМЕЧАНИЕ 1 . Множество функций $k$-значной логики Поста $P_{k}$, где $k \in\{2,3, \ldots\}$, есть множество $\left\{f\left(x_{1}, \ldots, x_{n}\right):\{0,1, \ldots\right.$, 
$\left.k-1\}^{n} \longmapsto\{0,1, \ldots, k-1\}\right\}$, где $\{0,1, \ldots, k-1\}^{n}$ есть декартова $n$-ая степень множества $\{0,1, \ldots, k-1\}$. В частности, множество функций $\mathrm{P}_{3}$ содержит все функции, аргументы которых, как и сами функции, принимают значения из множества $\{0,1,2\}$. В настоящей работе приняты иные обозначения для элементов множества значений функций и их аргументов: значение 0 остается без изменений; вместо значения 1 пишем $1 / 2$; вместо значения 2, используем 1 .

ЗАМЕЧАНИЕ 2. С понятием предполноты непосредственно связано определяемое индуктивно понятие глубины.

ЗАМЕЧАНИЕ 3.

- Класс функций, соответствующий $k$-значной логике Поста $\mathrm{P}_{k}$, имеет глубину $d\left(P_{k}\right)$, равную нулю;

- Если класс $F \subseteq P_{k}$ имеет глубину $d(F)$, равную $n$, и класс $G$ предполон в $F$, то $d(G)=n+1$.

$\mathrm{B}$ частности, любой предполный в $\mathrm{P}_{k}$ класс имеет глубину $d(F)=1$.

\section{2 Класс функций, соответствующий трехзначной логике Лукасевича}

Самой известной и исторически первой неклассической многозначной логикой является трехзначная логика Лукасевича $L_{3}$. Дальнейшие результаты имеют отношение к данной логике, поэтому имеет смысл рассмотреть ее специально. В данном разделе будет также доказано утверждение о том, что множество функций логики Лукасевича является предполным в трехзначной логике Поста и совпадает с множеством функций, сохраняющих неравенство промежуточного значения в $P_{3}$, а именно: $L_{3}=\left\{f\left(x_{1}, \ldots, x_{n}\right) \in P_{3}: \forall\left\langle\alpha_{1}, \ldots, \alpha_{n}\right\rangle\left(\neg \exists i \in\{1,2, \ldots, n\}\left(\alpha_{i}=\right.\right.\right.$ $\left.\left.1 / 2) \Rightarrow f\left(\alpha_{1}, \ldots, \alpha_{n}\right) \neq 1 / 2\right)\right\}$. Множество функций логики Лукасевича может быть определено как замыкание системы функций $\{\neg L x, \mathrm{x} \rightarrow y\}$, где $\neg L x=1-\mathrm{x}$, а функция $\mathrm{x} \rightarrow y$, называемая импликацией Лукасевича, определятся таблично: 


\begin{tabular}{|c|c|c|c|}
\hline$\rightarrow$ & 1 & $1 / 2$ & 0 \\
\hline 1 & 1 & $1 / 2$ & 0 \\
\hline $1 / 2$ & 1 & 1 & $1 / 2$ \\
\hline 0 & 1 & 1 & 1 \\
\hline
\end{tabular}

Заметим, что существуют и другие системы функций, полные в $L_{3}$. В частности, в дальнейшем будут использоваться следующие полные в $L_{3}$ системы функций: $\left\{\neg{ }_{L} x, \square \mathrm{x}, x \vee y\right\},\left\{\neg_{L} x, \diamond \mathrm{x}, x \vee\right.$ $y\}$ и $\{\neg L x, \nabla \mathrm{x}, x \vee y\}$, - где функция $\square$ х называется оператором необходимости, функция $\diamond$ х называется оператором возможности, а функция $\nabla$ х называется оператором случайности (см. [6, разд. 2.1.2]). Функция $x \vee y$ есть $\max (x, y)$. Дадим табличные определения операторов необходимости, возможности и случайности:

\begin{tabular}{|c|c|c|c|}
\hline$x$ & $\square \mathrm{x}$ & $\diamond \mathrm{x}$ & $\nabla \mathrm{x}$ \\
\hline 1 & 1 & 1 & 0 \\
\hline $1 / 2$ & 0 & 1 & 1 \\
\hline 0 & 0 & 0 & 0 \\
\hline
\end{tabular}

Очевидно, что все вышеприведенные функции удовлетворяют условию сохранения неравенства промежуточного значения, а операция суперпозиции сохраняет свойство удовлетворения данному условию. Покажем теперь, что добавление к полной в $L_{3}$ системе функций функции из $P_{3}$, не сохраняющей неравенства промежуточного значения, дает систему, полную в $\mathrm{P}_{3}$.

Для этого используем утверждение, содержащееся в [5], а именно: добавление к функциям из $L_{3}$ функции $\mathrm{T}(\mathrm{x})$, принимающей значение $1 / 2$ на всех значениях х, дает систему, полную в $\mathrm{P}_{3}$. Функция $\mathrm{T}(\mathrm{x})$ называется оператором Слупецкого.

Рассмотрим функцию $f\left(x_{1}, \ldots, x_{n}\right) \in \mathrm{P}_{3}$, такую, что:

$\exists\left\langle\alpha_{1}, \ldots, \alpha_{n}\right\rangle\left(\neg \exists i \in\{1,2, \ldots, n\}\left(\alpha_{i}=1 / 2\right) \& f\left(\alpha_{1}, \ldots, \alpha_{n}\right)=1 / 2\right)$.

Поскольку $L_{3}$ содержит константы 1 и 0 , может быть осуществлена подстановка соответствующих констант вместо переменных $x_{i}(1 \leq i \leq n)$ в $f\left(x_{1}, \ldots, x_{n}\right)$ в зависимости от того, чему равны соответствующие значения $\alpha_{i}$ в $\left\langle\alpha_{1}, \ldots, \alpha_{n}\right\rangle$. В результате получим функцию, тождественно равную промежуточному значению. Следовательно, $\left[L_{3} \cup f\left(x_{1}, \ldots, x_{n}\right)\right]=\mathrm{P}_{3}$, что и требовалось доказать. 


\section{3 Класс $\mathrm{K}_{3}^{D}$ диагональных функций}

Докажем теперь предполноту класса диагональных функций $\mathrm{K}_{3}^{D}$ в $L_{3}$. Для доказательства потребуется использовать функции сильной логики Клини $\mathrm{K}_{3}$. Базовыми в $\mathrm{K}_{3}$ являются функции $\left\{\neg_{L} x, x \vee y\right\}$, где, как и прежде, $x \vee y=\max (x, y)$, а $\neg{ }_{L} x=$ $1-\mathrm{x}$.

Класс $\mathrm{K}_{3}^{D}$ определяется следующим образом:

$\mathrm{K}_{3}^{D}=\left\{f\left(x_{1}, \ldots, x_{n}\right) \in L_{3}: \forall\left\langle\alpha_{1}, \ldots, \alpha_{n}\right\rangle,\left\langle\beta_{1}, \ldots, \beta_{n}\right\rangle\right.$

$\left(\left(\exists i \in\{1,2, \ldots, n\}\left(\alpha_{i}=1 / 2\right) \Rightarrow\left(f\left(\alpha_{1}, \ldots, \alpha_{n}\right)=1 \Rightarrow\right.\right.\right.$

$\left.\left.\forall\left\langle\alpha_{1}^{\prime}, \ldots, \alpha_{n}^{\prime}\right\rangle\left(f\left(\alpha_{1}^{\prime}, \ldots, \alpha_{n}^{\prime}\right)=1\right)\right)\right) \&\left(\exists j \in\{1,2, \ldots, n\}\left(\beta_{j}=\right.\right.$ $1 / 2) \Rightarrow$

$\left.\left.\left.\left(f\left(\beta_{1}, \ldots, \beta_{n}\right)=0 \Rightarrow \forall\left\langle\beta_{1}^{\prime}, \ldots, \beta_{n}^{\prime}\right\rangle\left(f\left(\beta_{1}^{\prime}, \ldots, \beta_{n}^{\prime}\right)=0\right)\right)\right)\right)\right\}$,

где $\left\langle\alpha_{1}^{\prime}, \ldots, \alpha_{n}^{\prime}\right\rangle,\left\langle\beta_{1}^{\prime}, \ldots, \beta_{n}^{\prime}\right\rangle$ есть результаты произвольных замен всех вхождений значения $1 / 2$ в $\left\langle\alpha_{1}, \ldots, \alpha_{n}\right\rangle$ и $\left\langle\beta_{1}, \ldots, \beta_{n}\right\rangle$, соответственно, на единицы и нули.

ЗАМЕЧАНИЕ 4. Предыдущее определение означает, что каждый из наборов значений $\left\langle\alpha_{1}, \ldots, \alpha_{n}\right\rangle,\left\langle\beta_{1}, \ldots, \beta_{n}\right\rangle$ порождает, возможно, пустой класс подстановок $\left\langle\alpha_{1}^{\prime}, \ldots, \alpha_{n}^{\prime}\right\rangle,\left\langle\beta_{1}^{\prime}, \ldots, \beta_{n}^{\prime}\right\rangle$ единиц и нулей в исходные наборы. Эти подстановки могут быть просто и естественно описаны при помощи введенного Ю.В. Ивлевым в [4] понятия квазифункции.

ОПРЕДЕЛЕНИЕ 6. Под квазифункцией будем понимать соответсвие, в силу которого определенный объект из некоторого множества соотносится с некоторым объектом из определенного подмножества того же самого множества.

Дадим табличное определение одноместной квазифункции $\varphi(x)$ :

\begin{tabular}{|c|c|}
\hline$x$ & $\varphi(x)$ \\
\hline 1 & 1 \\
\hline $1 / 2$ & $1 / 0$ \\
\hline 0 & 0 \\
\hline
\end{tabular}

Здесь запись $1 / 0$ означает, что значению $1 / 2$ ставится в соответствие 1 или 0 . С использованием данной квазифункции определение класса $\mathrm{K}_{3}^{D}$ может быть записано в следующем виде:

$\mathrm{K}_{3}^{D}=\left\{f\left(x_{1}, \ldots, x_{n}\right) \in L_{3}: \forall\left\langle\alpha_{1}, \ldots, \alpha_{n}\right\rangle((\exists i \in\{1,2, \ldots, n\}\right.$ $\left.\left(\alpha_{i}=1 / 2\right)\right) \Rightarrow\left(\left(f\left(\alpha_{1}, \ldots, \alpha_{n}\right) \in\{1,0\}\right) \Rightarrow\left(f\left(\alpha_{1}, \ldots, \alpha_{n}\right)=\right.\right.$ $\left.\left.\left.\left.f\left(\varphi\left(\alpha_{1}\right), \ldots, \varphi\left(\alpha_{n}\right)\right)\right)\right)\right)\right\}$. 
ЗАМЕЧАНИЕ 5. Классу $\mathrm{K}_{3}^{D}$ принадлежат все функции сильной регулярной логики Клини $\mathrm{K}_{3}$, а также константы 1 и 0 , т. е. $\left[K_{3} \cup\{1,0\}\right] \subset \mathrm{K}_{3}^{D}$. Однако $\mathrm{K}_{3}^{D}$ не принадлежит ни одна из функций $\square \mathrm{x}, \diamond \mathrm{x}, \nabla \mathrm{x}$, а также импликация Лукасевича $x \rightarrow y$. Следовательно, класс диагональных функций не совпадает с классом функций логики Лукасевича. Данный класс содержит и функции, не являющиеся регулярными, по Клини. Примером такой функции является $\psi(x, y)$, определяемая следующей таблицей:

\begin{tabular}{|c|c|c|c|}
\hline$\psi$ & 1 & $1 / 2$ & 0 \\
\hline 1 & 1 & $1 / 2$ & 1 \\
\hline $1 / 2$ & $1 / 2$ & 1 & $1 / 2$ \\
\hline 0 & 1 & $1 / 2$ & 1 \\
\hline
\end{tabular}

Докажем, что $\mathrm{K}_{3}^{D}$ является замкнутым. Для этого достаточно показать, что если функции $f\left(x_{1}, \ldots, x_{s}\right), f_{1}\left(x_{11}, x_{21}, \ldots, x_{n 1}\right)$, $f_{2}\left(x_{12}, x_{22}, \ldots, x_{n 2}\right), \ldots, f_{s}\left(x_{1 s}, x_{2 s}, \ldots, x_{n s}\right)$ принадлежат $\mathrm{K}_{3}^{D}$, то и функция $\Phi=f\left(f_{1}\left(x_{11}, x_{21}, \ldots, x_{n 1}\right), f_{2}\left(x_{12}, x_{22}, \ldots, x_{n 2}\right), \ldots\right.$, $\left.f_{s}\left(x_{1 s}, x_{2 s}, \ldots, x_{n s}\right)\right)$ также принадлежит $\mathrm{K}_{3}^{D}$. Поскольку функции $f\left(x_{1}, \ldots, x_{s}\right), f_{1}\left(x_{11}, x_{21}, \ldots, x_{n 1}\right), f_{2}\left(x_{12}, x_{22}, \ldots, x_{n 2}\right), \ldots$, $f_{s}\left(x_{1 s}, x_{2 s}, \ldots, x_{n s}\right)$ принадлежат $L_{3}$, то на всех наборах $\left\langle\alpha_{11}, \alpha_{21}\right.$, $\left.\ldots, \alpha_{n 1}, \alpha_{12}, \alpha_{22}, \ldots, \alpha_{n 2}, \ldots, \alpha_{1 s}, \alpha_{2 s}, \ldots, \alpha_{n s}\right\rangle$ и $\left\langle\beta_{11}, \beta_{21}, \ldots, \beta_{n 1}\right.$, $\left.\beta_{12}, \beta_{22}, \ldots, \beta_{n 2}, \ldots, \beta_{1 s}, \beta_{2 s}, \ldots, \beta_{n s}\right\rangle$ значений переменных $x_{11}$, $x_{21}, \ldots, x_{n 1}, x_{12}, x_{22}, \ldots, x_{n 2}, \ldots, x_{1 s}, x_{2 s}, \ldots, x_{n s}$, не содержащих вхождений промежуточного значения, функция $\Phi$, очевидно, удовлетворяет определению $\mathrm{K}_{3}^{D}$.

Если же в наборе значений переменных функции $\Phi$ имеются вхождения промежуточного значения, то $\Phi$ либо принимает промежуточное значение, либо значение $\Phi$ принадлежит множеству $\{1,0\}$. В первом случае функция $\Phi$ удовлетворяет условиям определения класса $\mathrm{K}_{3}^{D}$. Во втором случае данная функция также удовлетворяет условиям определения, поскольку для каждой из функций $f_{j}(1 \leq j \leq s)$ будет иметь место один из следующих случаев:

1. Если значение функции $f_{j}$ на некотором наборе значений переменных равняется $1 / 2$, то на любых соответствующих поднаборах $\left\langle\alpha_{1 j}^{\prime}, \ldots, \alpha_{n j}^{\prime}\right\rangle$ и $\left\langle\beta_{1 j}^{\prime}, \ldots, \beta_{n j}^{\prime}\right\rangle$ значений переменных $x_{1 j}, x_{2 j}, \ldots, x_{n j}$ значение $f_{j}$ принадлежит $\{1,0\}$. 
Однако поскольку $f\left(x_{1}, \ldots, x_{s}\right)$ принадлежит $\mathrm{K}_{3}^{D}$, то и функция $\Phi$ не изменит в этом случае своего значения.

2. Если $f_{j}$ принадлежит множеству $\{1,0\}$, то так как $f_{j} \in$ $\mathrm{K}_{3}^{D}$, значение данной функции не изменится на любых соответствующих поднаборах $\left\langle\alpha_{1 j}^{\prime}, \ldots, \alpha_{n j}^{\prime}\right\rangle$ и $\left\langle\beta_{1 j}^{\prime}, \ldots, \beta_{n j}^{\prime}\right\rangle$ значений переменных $x_{1 j}, x_{2 j}, \ldots, x_{n j}$, а следовательно, значение функции $\Phi$ не изменится и в этом случае.

Следовательно, функция $\Phi$ также принадлежит $\mathrm{K}_{3}^{D}$, что и требовалось доказать.

TEOРЕМА 1. Класс диагональных функций $\mathrm{K}_{3}^{D}$ является предполным в $L_{3}$.

Доказательство. Добавим к функциям $\mathrm{K}_{3}^{D}$ функцию $f\left(x_{1}, \ldots\right.$, $\left.x_{n}\right) \in L_{3}$, такую что $f\left(x_{1}, \ldots, x_{n}\right) \notin \mathrm{K}_{3}^{D}$. Поскольку $f\left(x_{1}, \ldots\right.$, $\left.x_{n}\right) \notin \mathrm{K}_{3}^{D}$, возможны случаи:

1. Существует набор $\left\langle\alpha_{1}, \ldots, \alpha_{n}\right\rangle$ значений переменных $x_{1}, \ldots$, $x_{n}$ такой, что в $\left\langle\alpha_{1}, \ldots, \alpha_{n}\right\rangle$ имеются вхождения промежуточного значения и $f\left(\alpha_{1}, \ldots, \alpha_{n}\right)=1$. А также существует набор $\left\langle\alpha_{1}^{\prime}, \ldots, \alpha_{n}^{\prime}\right\rangle$, в котором все вхождения промежуточного значения в $\left\langle\alpha_{1}, \ldots, \alpha_{n}\right\rangle$ заменены на единицы и нули, такой, что $f\left(\alpha_{1}^{\prime}, \ldots\right.$, $\left.\alpha_{n}^{\prime}\right) \neq 1$. Так как $f\left(x_{1}, \ldots, x_{n}\right)$ принадлежит множеству функций логики Лукасевича, то $f\left(\alpha_{1}^{\prime}, \ldots, \alpha_{n}^{\prime}\right) \neq 1 / 2$, поскольку в $\left\langle\alpha_{1}^{\prime}, \ldots, \alpha_{n}^{\prime}\right\rangle$ не имеется вхождений значения $1 / 2$. Следовательно, $f\left(\alpha_{1}^{\prime}, \ldots, \alpha_{n}^{\prime}\right)=0$. Осуществим теперь подстановку соответствующих констант вместо всех $\alpha_{i}(1 \leq i \leq n)$, принадлежащих множеству $\{1,0\}$ в $\left\langle\alpha_{1}, \ldots, \alpha_{n}\right\rangle$, в функцию $f\left(x_{1}, \ldots, x_{n}\right)$. На место незатронутых предыдущей подстановкой переменных в $f\left(x_{1}, \ldots, x_{n}\right)$ подставим функции $\mathrm{x}$ и $\neg L$ х в зависимости от того, какие константы (1 или 0 соответственно) были подставлены в $\left\langle\alpha_{1}^{\prime}, \ldots, \alpha_{n}^{\prime}\right\rangle$ вместо каждого из вхождений значения $1 / 2$ в $\left\langle\alpha_{1}, \ldots, \alpha_{n}\right\rangle$. Такие подстановки могут быть осуществлены, поскольку $\mathrm{K}_{3}^{D}$ содержит функции 1,0 и $\neg L \mathrm{x}$.

В результате получим функцию $f^{\prime}(x)$, зависящую от одной переменной, такую, что $f^{\prime}(1 / 2)=1$ и $f^{\prime}(1) \neq 1$ или $f^{\prime}(0) \neq 1$. Следовательно, $f^{\prime}(x)$ совпадает с одной из трех функций: 


\begin{tabular}{|c|c|}
\hline$x$ & $f_{1}^{\prime}(x)$ \\
\hline 1 & 0 \\
\hline $1 / 2$ & 1 \\
\hline 0 & 1 \\
\hline
\end{tabular}

\begin{tabular}{|c|c|}
\hline$x$ & $f_{2}^{\prime}(x)$ \\
\hline 1 & 1 \\
\hline $1 / 2$ & 1 \\
\hline 0 & 0 \\
\hline
\end{tabular}

\begin{tabular}{|c|c|}
\hline$x$ & $f_{3}^{\prime}(x)$ \\
\hline 1 & 0 \\
\hline $1 / 2$ & 1 \\
\hline 0 & 0 \\
\hline
\end{tabular}

Функции $f_{2}^{\prime}(\mathrm{x})$ и $f_{3}^{\prime}(\mathrm{x})$ совпадают с операторами $\diamond_{\mathrm{x}}$ и $\nabla \mathrm{x}$, соответственно. Функция $\neg L f_{1}^{\prime}(\mathrm{x})$ эквивалентна оператору $\square \mathrm{x}$. Так как класс $K_{3}^{D}$ содержит функции $\neg L$ х и $x \vee y$, а системы $\{\neg L \mathrm{x}, \square \mathrm{x}$,

$x \vee y\},\left\{\neg_{L} \mathrm{x}, \diamond \mathrm{x}, x \vee y\right\}$ и $\left\{\neg_{L} \mathrm{x}, \nabla \mathrm{x}, x \vee y\right\}$ являются полными в $L_{3}$ (см. [5, разд. 2.1.2]), то $\left[K_{3}^{D} \cup f\left(x_{1}, \ldots, x_{n}\right)\right]=L_{3}$.

Рассмотрение случая 1 завершено.

2. Существует набор $\left\langle\beta_{1}, \ldots, \beta_{n}\right\rangle$ значений переменных $x_{1}, \ldots$, $x_{n}$ такой, что в $\left\langle\beta_{1}, \ldots, \beta_{n}\right\rangle$ имеются вхождения промежуточного значения и $f\left(\beta_{1}, \ldots, \beta_{n}\right)=0$. А также существует набор $\left\langle\beta_{1}^{\prime}, \ldots, \beta_{n}^{\prime}\right\rangle$, в котором все вхождения промежуточного значения в $\left\langle\beta_{1}, \ldots, \beta_{n}\right\rangle$ заменены на единицы и нули, такой, что $f\left(\beta_{1}^{\prime}, \ldots\right.$, $\left.\beta_{n}^{\prime}\right) \neq 0$. Так как $f\left(x_{1}, \ldots, x_{n}\right)$ принадлежит множеству функций логики Лукасевича, то $f\left(\beta_{1}^{\prime}, \ldots, \beta_{n}^{\prime}\right) \neq 1 / 2$, поскольку в $\left\langle\beta_{1}^{\prime}, \ldots, \beta_{n}^{\prime}\right\rangle$ не имеется вхождений значения $1 / 2$. Следовательно, $f\left(\beta_{1}^{\prime}, \ldots, \beta_{n}^{\prime}\right)=1$. Осуществим теперь подстановку соответствующих констант вместо всех $\beta_{j}(1 \leq j \leq n)$, принадлежащих множеству $\{1,0\}$ в $\left\langle\beta_{1}, \ldots, \beta_{n}\right\rangle$, в функцию $f\left(x_{1}, \ldots, x_{n}\right)$. На место незатронутых предыдущей подстановкой переменных в $f\left(x_{1}, \ldots, x_{n}\right)$ подставим функции $\mathrm{x}$ и $\neg L \mathrm{x}$ в зависимости от того, какие константы (1 или 0 соответственно) были подставлены в $\left\langle\beta_{1}^{\prime}, \ldots, \beta_{n}^{\prime}\right\rangle$ вместо каждого из вхождений значения $1 / 2$ в $\left\langle\beta_{1}, \ldots, \beta_{n}\right\rangle$. Такие подстановки могут быть осуществлены, поскольку $\mathrm{K}_{3}^{D}$ содержит функции 1,0 и $\neg L \mathrm{x}$.

В результате получим функцию $f^{\prime}(x)$, зависящую от одной переменной, такую, что $f^{\prime}(1 / 2)=0$ и $f^{\prime}(1) \neq 0$ или $f^{\prime}(0) \neq 0$. Следовательно, $f^{\prime}(x)$ совпадает с одной из трех функций:

\begin{tabular}{|c|c|}
\hline$x$ & $f_{1}^{\prime}(x)$ \\
\hline 1 & 1 \\
\hline $1 / 2$ & 0 \\
\hline 0 & 0 \\
\hline
\end{tabular}

\begin{tabular}{|c|c|}
\hline$x$ & $f_{2}^{\prime}(x)$ \\
\hline 1 & 0 \\
\hline $1 / 2$ & 0 \\
\hline 0 & 1 \\
\hline
\end{tabular}

\begin{tabular}{|c|c|}
\hline$x$ & $f_{3}^{\prime}(x)$ \\
\hline 1 & 1 \\
\hline $1 / 2$ & 0 \\
\hline 0 & 1 \\
\hline
\end{tabular}

Функция $f_{1}^{\prime}(\mathrm{x})$ эквивалентна оператору $\square \mathrm{x}$. А функции $\neg L f_{2}^{\prime}(\mathrm{x})$ 
и $\neg_{L} f_{3}^{\prime}(\mathrm{x})$ совпадают с операторами $\diamond \mathrm{x}$ и $\nabla \mathrm{x}$ соответственно. Так как класс $K_{3}^{D}$ содержит функции $\neg_{L} \mathrm{x}$ и $x \vee y$, а системы $\left\{\neg_{L} \mathrm{x}, \square \mathrm{x}, x \vee y\right\},\left\{\neg_{L} \mathrm{x}, \diamond \mathrm{x}, x \vee y\right\}$ и $\left\{\neg_{L} \mathrm{x}, \nabla \mathrm{x}, x \vee y\right\}$ являются полными в $L_{3}$ (см. [5, разд. 2.1.2]), то $\left[K_{3}^{D} \cup f\left(x_{1}, \ldots, x_{n}\right)\right]=L_{3}$.

Рассмотрение случая 2 завершено.

Доказательство теоремы завершено.

Q.E.D.

Таким образом, установлено, что класс диагональных функций является предполным в классе функций трехзначной логики Лукасевича.

\section{4 Класс $\mathrm{H}_{3}^{*}$, двойственный трехзначной логике Гейтинга}

В [7] было показано, что класс функций трехзначной логики Гейтинга $\mathrm{H}_{3}$ является предполным в $L_{3}$. В этом разделе будет доказана теорема о том, что и класс $\mathrm{H}_{3}^{*}$, двойственный $\mathrm{H}_{3}$, также предполон в $L_{3}$. В доказательстве предполноты $\mathrm{H}_{3}$ существенным образом используется тот факт, что множество функций данного класса совпадает с множеством функций, дистрибутивных относительно функции $\neg \neg \mathrm{x}$, где $\neg x$ эквивалентно функции $\neg_{L} \diamond x$ логики Лукасевича. А именно: $\mathrm{H}_{3}=\left\{f\left(x_{1}, \ldots, x_{n}\right) \in\right.$ $\left.L_{3}: \neg \neg f\left(x_{1}, \ldots, x_{n}\right)=f\left(\neg \neg x_{1}, \ldots, \neg \neg x_{n}\right)\right\}$. Достаточно заметить, что $\neg \neg \mathrm{x}=\neg_{L} \diamond \neg_{L} \diamond x=\square \diamond x=\diamond x$. Тогда приведенное определение можно заменить эквивалентным ему:

$$
\mathrm{H}_{3}=\left\{f\left(x_{1}, \ldots, x_{n}\right) \in L_{3}: \diamond f\left(x_{1}, \ldots, x_{n}\right)=f\left(\diamond x_{1}, \ldots, \diamond x_{n}\right)\right\}
$$

Таким образом, двойственный $\boldsymbol{\kappa} \mathrm{H}_{3} \boldsymbol{\kappa} \boldsymbol{л л а с с ~ м о ж е т ~ б ы т ь ~ о п р е - ~}$ делен следующим способом ${ }^{1}$ :

$$
\mathrm{H}_{3}^{*}=\left\{f\left(x_{1}, \ldots, x_{n}\right) \in L_{3}: \square f\left(x_{1}, \ldots, x_{n}\right)=f\left(\square x_{1}, \ldots, \square x_{n}\right)\right\}
$$

Докажем, что класс $\mathrm{H}_{3}^{*}$ является замкнутым. Для этого достаточно показать, что если функции $f\left(x_{1}, \ldots, x_{s}\right), f_{1}\left(x_{11}, x_{21}, \ldots\right.$, $\left.x_{n 1}\right), f_{2}\left(x_{12}, x_{22}, \ldots, x_{n 2}\right), \ldots, f_{s}\left(x_{1 s}, x_{2 s}, \ldots, x_{n s}\right)$ принадлежат $\mathrm{H}_{3}^{*}$, то и функция $\Phi=f\left(f_{1}\left(x_{11}, x_{21}, \ldots, x_{n 1}\right), f_{2}\left(x_{12}, x_{22}, \ldots, x_{n 2}\right)\right.$, $\left.\ldots, f_{s}\left(x_{1 s}, x_{2 s}, \ldots, x_{n s}\right)\right)$ также принадлежит $\mathrm{H}_{3}^{*}$.

\footnotetext{
1 Заметим, что класс $\mathrm{H}_{3}^{*}$, по всей видимости, совпадает с классом функций логики $G_{3}^{*}$, впервые рассмотренной А. С. Карпенко в [5] под названием трехзначной логики Брауэра.
} 
Так как функция $f\left(x_{1}, \ldots, x_{s}\right)$ принадлежит $\mathrm{H}_{3}^{*}$, то $\square \Phi=$ $f\left(\square f_{1}\left(x_{11}, x_{21}, \ldots, x_{n 1}\right), \quad \square f_{2}\left(x_{12}, x_{22}, \ldots, x_{n 2}\right), \ldots, \quad \square f_{s}\left(x_{1 s}, x_{2 s}\right.\right.$, $\left.\left.\ldots, x_{n s}\right)\right)$. Поскольку каждая из функций $f_{j}(1 \leq j \leq s)$ принадлежит $\mathrm{H}_{3}^{*}$, то для них выполняются равенства $\square f_{j}\left(x_{1 j}, x_{2 j}, \ldots\right.$, $\left.x_{n j}\right)=f_{j}\left(\square x_{1 j}, \square x_{2 j}, \ldots, \square x_{n j}\right)$. Следовательно, $\square \Phi=f\left(f_{1}\left(\square x_{11}\right.\right.$, $\left.\square x_{21}, \ldots, \square x_{n 1}\right), f_{2}\left(\square x_{12}, \square x_{22}, \ldots, \square x_{n 2}\right), \ldots, f_{s}\left(\square x_{1 s}, \square x_{2 s}, \ldots\right.$, $\left.\left.\square x_{n s}\right)\right)$.

Значит, функция $\Phi$ также принадлежит $\mathrm{H}_{3}^{*}$, что и требовалось доказать.

Для доказательства предполноты $\mathrm{H}_{3}^{*}$ потребуется использовать функции трехзначной логики Брауэра $G_{3}^{*}$. Исходными в логике Брауэра являются функции $\left\{\neg G_{3}^{*} x, x \& y, x \vee y, x \Leftarrow y\right\}$, где $x \& y=\min (x, y), x \vee y=\max (x, y)$, а функции $\neg G_{3}^{*} x$ и $x \Leftarrow y$ определяются следующими таблицами:

\begin{tabular}{|c|c|}
\hline$x$ & $\neg G_{3}^{*} x$ \\
\hline 1 & 0 \\
\hline $1 / 2$ & 1 \\
\hline 0 & 1 \\
\hline
\end{tabular}

\begin{tabular}{|c|c|c|c|}
\hline$\Leftarrow$ & 1 & $1 / 2$ & 0 \\
\hline 1 & 0 & 0 & 0 \\
\hline $1 / 2$ & 1 & 0 & 0 \\
\hline 0 & 1 & $1 / 2$ & 0 \\
\hline
\end{tabular}

$\mathrm{H}_{3}^{*}$ содержит функцию $\square \mathrm{x}$, в силу очевидного равенства $\square \square \mathrm{x}=$ $\square \square$. Покажем, что все исходные функции $G_{3}^{*}$ принадлежат $\mathrm{H}_{3}^{*}$, то есть $G_{3}^{*} \subset \mathrm{H}_{3}^{*}$. Для этого построим истинностные таблицы для каждой из функций, входящих в следующие пары:

1. $\square \neg G_{3}^{*} x, \neg G_{3}^{*} \square x$;

2. $\square(x \& y), \square x \& \square y$;

3. $\square(x \vee y), \square x \vee \square y$;

4. $\square(x \Leftarrow y), \square x \Leftarrow \square y$

Приведем здесь в качестве примера таблицы всех пар функций.

1.

\begin{tabular}{|c|c|c|}
\hline$\square$ & $\neg G_{3}^{*}$ & $x$ \\
\hline 0 & 0 & 1 \\
\hline 1 & 1 & $1 / 2$ \\
\hline 1 & 1 & 0 \\
\hline
\end{tabular}

\begin{tabular}{|c|c|c|}
\hline$\neg G_{3}^{*}$ & $\square$ & $x$ \\
\hline 0 & 1 & 1 \\
\hline 1 & 0 & $1 / 2$ \\
\hline 1 & 0 & 0 \\
\hline
\end{tabular}


2.

\begin{tabular}{|c|c|c|c|}
\hline$\square$ & $(x$ & $\&$ & $y)$ \\
\hline 1 & 1 & 1 & 1 \\
\hline 0 & 1 & $1 / 2$ & $1 / 2$ \\
\hline 0 & 1 & 0 & 0 \\
\hline 0 & $1 / 2$ & $1 / 2$ & 1 \\
\hline 0 & $1 / 2$ & $1 / 2$ & $1 / 2$ \\
\hline 0 & $1 / 2$ & 0 & 0 \\
\hline 0 & 0 & 0 & 1 \\
\hline 0 & 0 & 0 & $1 / 2$ \\
\hline 0 & 0 & 0 & 0 \\
\hline
\end{tabular}

\begin{tabular}{|c|c|c|c|c|}
\hline$\square$ & $x$ & $\&$ & $\square$ & $y$ \\
\hline 1 & 1 & 1 & 1 & 1 \\
\hline 1 & 1 & 0 & 0 & $1 / 2$ \\
\hline 1 & 1 & 0 & 0 & 0 \\
\hline 0 & $1 / 2$ & 0 & 1 & 1 \\
\hline 0 & $1 / 2$ & 0 & 0 & $1 / 2$ \\
\hline 0 & $1 / 2$ & 0 & 0 & 0 \\
\hline 0 & 0 & 0 & 1 & 1 \\
\hline 0 & 0 & 0 & 0 & $1 / 2$ \\
\hline 0 & 0 & 0 & 0 & 0 \\
\hline
\end{tabular}

3.

\begin{tabular}{|c|c|c|c|}
\hline$\square$ & $(x$ & $\vee$ & $y)$ \\
\hline 1 & 1 & 1 & 1 \\
\hline 1 & 1 & 1 & $1 / 2$ \\
\hline 1 & 1 & 1 & 0 \\
\hline 1 & $1 / 2$ & 1 & 1 \\
\hline 0 & $1 / 2$ & $1 / 2$ & $1 / 2$ \\
\hline 0 & $1 / 2$ & $1 / 2$ & 0 \\
\hline 1 & 0 & 1 & 1 \\
\hline 0 & 0 & $1 / 2$ & $1 / 2$ \\
\hline 0 & 0 & 0 & 0 \\
\hline
\end{tabular}

\begin{tabular}{|c|c|c|c|c|}
\hline$\square$ & $x$ & $\vee$ & $\square$ & $y$ \\
\hline 1 & 1 & 1 & 1 & 1 \\
\hline 1 & 1 & 1 & 0 & $1 / 2$ \\
\hline 1 & 1 & 1 & 0 & 0 \\
\hline 0 & $1 / 2$ & 1 & 1 & 1 \\
\hline 0 & $1 / 2$ & 0 & 0 & $1 / 2$ \\
\hline 0 & $1 / 2$ & 0 & 0 & 0 \\
\hline 0 & 0 & 1 & 1 & 1 \\
\hline 0 & 0 & 0 & 0 & $1 / 2$ \\
\hline 0 & 0 & 0 & 0 & 0 \\
\hline
\end{tabular}

4.

\begin{tabular}{|c|c|c|c|}
\hline$\square$ & $(x$ & $\Leftarrow$ & $y)$ \\
\hline 0 & 1 & 0 & 1 \\
\hline 0 & 1 & 0 & $1 / 2$ \\
\hline 0 & 1 & 0 & 0 \\
\hline 1 & $1 / 2$ & 1 & 1 \\
\hline 0 & $1 / 2$ & 0 & $1 / 2$ \\
\hline 0 & $1 / 2$ & 0 & 0 \\
\hline 1 & 0 & 1 & 1 \\
\hline 0 & 0 & $1 / 2$ & $1 / 2$ \\
\hline 0 & 0 & 0 & 0 \\
\hline
\end{tabular}

\begin{tabular}{|c|c|c|c|c|}
\hline$\square$ & $x$ & $\Leftarrow$ & $\square$ & $y$ \\
\hline 1 & 1 & 0 & 1 & 1 \\
\hline 1 & 1 & 0 & 0 & $1 / 2$ \\
\hline 1 & 1 & 0 & 0 & 0 \\
\hline 0 & $1 / 2$ & 1 & 1 & 1 \\
\hline 0 & $1 / 2$ & 0 & 0 & $1 / 2$ \\
\hline 0 & $1 / 2$ & 0 & 0 & 0 \\
\hline 0 & 0 & 1 & 1 & 1 \\
\hline 0 & 0 & 0 & 0 & $1 / 2$ \\
\hline 0 & 0 & 0 & 0 & 0 \\
\hline
\end{tabular}

Перейдем теперь к доказательству основной теоремы этого раздела.

TЕОРЕМА 2. Класс $\mathrm{H}_{3}^{*}$ является предполным в $L_{3}$. 
Доказательство. Рассмотрим функцию $f\left(x_{1}, \ldots, x_{n}\right) \in L_{3}$ такую, что $f\left(x_{1}, \ldots, x_{n}\right) \notin \mathrm{H}_{3}^{*}$. Заметим, что $\mathrm{H}_{3}^{*}$ содержит функции $\{\square x, x \vee y\}$. Следовательно, для доказательства утверждения теоремы достаточно показать, что $\neg_{L} x \in\left[\mathrm{H}_{3}^{*} \cup f\left(x_{1}, \ldots, x_{n}\right)\right]$, так как система функций $\left\{\neg{ }_{L} x, \square x, x \vee y\right\}$ является полной в $L_{3}$. Поскольку $f\left(x_{1}, \ldots, x_{n}\right) \notin \mathrm{H}_{3}^{*}$, то существует набор $\left\langle\alpha_{1}, \ldots, \alpha_{n}\right\rangle$ значений переменных $x_{1}, \ldots, x_{n}$, такой, что $\square f\left(\alpha_{1}, \ldots, \alpha_{n}\right) \neq$ $f\left(\square \alpha_{1}, \ldots, \square \alpha_{n}\right)$. Так как $\square 1=1$ и $\square 0=0$, и $f\left(x_{1}, \ldots, x_{n}\right) \in L_{3}$, то в $\left\langle\alpha_{1}, \ldots, \alpha_{n}\right\rangle$ имеются вхождения промежуточного значения. Осуществим подстановку переменной $x$, вместо всех вхождений промежуточного значения в $f\left(\alpha_{1}, \ldots, \alpha_{n}\right)$. На место переменных, не затронутых предыдущей подстановкой в $f\left(x_{1}, \ldots, x_{n}\right)$, подставим константы 1 или 0 в зависимости от того, чему равны соответствующие $\alpha_{j} \in\{1,0\}(1 \leq j \leq n)$ в $\left\langle\alpha_{1}, \ldots, \alpha_{n}\right\rangle$. Такие подстановки могут быть осуществлены, поскольку константы 1 и 0 принадлежат классу $\mathrm{H}_{3}^{*}$. В результате получим функцию $f^{\prime}(x)$, зависящую от одной переменной, такую, что: a) $f^{\prime}(1 / 2)=f\left(\alpha_{1}, \ldots, \alpha_{n}\right)$; и б) $f^{\prime}(0)=f\left(\square \alpha_{1}, \ldots, \square \alpha_{n}\right)$ (так как $\square 1 / 2=0$, а $\square 1=1$ и $\square 0=0$ ). Поскольку функция $\square \mathrm{x}$ удовлетворяет условию $\forall x, y(x=y \Rightarrow \square x=\square y)$, то из а) следует, что $\square f^{\prime}(1 / 2)=\square f\left(\alpha_{1}, \ldots, \alpha_{n}\right)$. Таким образом, получаем $\square f^{\prime}(1 / 2)=\square f\left(\alpha_{1}, \ldots, \alpha_{n}\right) \neq f\left(\square \alpha_{1}, \ldots, \square \alpha_{n}\right)=f^{\prime}(0)$, и отсюда $\square f^{\prime}(1 / 2) \neq f^{\prime}(0)$. Следовательно, $f^{\prime}(x)$ совпадает с одной из шести функций $f_{1}^{\prime}(x), \ldots, f_{6}^{\prime}(x)$ :

\begin{tabular}{|c|c|c|c|c|c|c|}
\hline$x$ & $f_{1}^{\prime}(x)$ & $f_{2}^{\prime}(x)$ & $f_{3}^{\prime}(x)$ & $f_{4}^{\prime}(x)$ & $f_{5}^{\prime}(x)$ & $f_{6}^{\prime}(x)$ \\
\hline 1 & 1 & 0 & 0 & 1 & 1 & 0 \\
\hline $1 / 2$ & $1 / 2$ & $1 / 2$ & 1 & 1 & 0 & 0 \\
\hline 0 & 1 & 1 & 0 & 0 & 1 & 1 \\
\hline
\end{tabular}

Покажем теперь, что функция $\neg_{L} x$ может быть получена с использованием каждой из функций $f_{1}^{\prime}(x), \ldots, f_{6}^{\prime}(x)$ и, быть может, других функций из $\mathrm{H}_{3}^{*}$. А именно покажем, что $\neg_{L} x=$ $\square x \Leftarrow f_{1}^{\prime}(x)=f_{2}^{\prime}(x)=\square x \Leftarrow\left(\neg G_{3}^{*} f_{3}^{\prime}(x) \vee x\right)=\square x \Leftarrow\left(\neg G_{3}^{*} f_{4}^{\prime}(x) \vee\right.$ $x)=\square x \Leftarrow\left(f_{5}^{\prime}(x) \vee x\right)=\square x \Leftarrow\left(f_{6}^{\prime}(x) \vee x\right)$. Приведем таблицы полученных функций:

\begin{tabular}{|c|c|c|c|c|}
\hline$\square$ & $x$ & $\Leftarrow$ & $f_{1}^{\prime}$ & $(x)$ \\
\hline 1 & 1 & 0 & 1 & 1 \\
\hline 0 & $1 / 2$ & $1 / 2$ & $1 / 2$ & $1 / 2$ \\
\hline 0 & 0 & 1 & 1 & 0 \\
\hline
\end{tabular}




\begin{tabular}{|c|c|c|c|c|c|c|c|}
\hline$\square$ & $x$ & $\Leftarrow$ & $\left(\neg G_{3}^{*}\right.$ & $f_{3}^{\prime}$ & $(x)$ & $\vee$ & $x)$ \\
\hline 1 & 1 & 0 & 1 & 0 & 1 & 1 & 1 \\
\hline 0 & $1 / 2$ & $1 / 2$ & 0 & 1 & $1 / 2$ & $1 / 2$ & $1 / 2$ \\
\hline 0 & 0 & 1 & 1 & 0 & 0 & 1 & 0 \\
\hline$\square$ & $x$ & $\Leftarrow$ & $\left(\neg G_{3}^{*}\right.$ & $f_{4}^{\prime}$ & $(x)$ & $\vee$ & $x)$ \\
\hline 1 & 1 & 0 & 0 & 1 & 1 & 1 & 1 \\
\hline 0 & $1 / 2$ & $1 / 2$ & 0 & 1 & $1 / 2$ & $1 / 2$ & $1 / 2$ \\
\hline 0 & 0 & 1 & 1 & 0 & 0 & 1 & 0 \\
\hline
\end{tabular}

\begin{tabular}{|c|c|c|c|c|c|c|}
\hline$\square$ & $x$ & $\Leftarrow$ & $\left(f_{5}^{\prime}\right.$ & $(x)$ & $\vee$ & $x)$ \\
\hline 1 & 1 & 0 & 1 & 1 & 1 & 1 \\
\hline 0 & $1 / 2$ & $1 / 2$ & 0 & $1 / 2$ & $1 / 2$ & $1 / 2$ \\
\hline 0 & 0 & 1 & 1 & 0 & 1 & 0 \\
\hline
\end{tabular}

\begin{tabular}{|c|c|c|c|c|c|c|}
\hline$\square$ & $x$ & $\Leftarrow$ & $\left(f_{6}^{\prime}\right.$ & $(x)$ & $\vee$ & $x)$ \\
\hline 1 & 1 & 0 & 0 & 1 & 1 & 1 \\
\hline 0 & $1 / 2$ & $1 / 2$ & 0 & $1 / 2$ & $1 / 2$ & $1 / 2$ \\
\hline 0 & 0 & 1 & 1 & 0 & 1 & 0 \\
\hline
\end{tabular}

Таким образом, теорема о предполноте класса $H_{3}^{*}$, двойственного трехзначной логике Гейтинга, в $L_{3}$ доказана.

Q.E.D.

\section{Литература}

[1] Гаврилов Г. П. О мощности множеств замкнутых классов конечной высоты в РА0 // ДАН. 1964. Т. 158. № 3. С. 503-506.

[2] Гаврилов Г. П., Яблонский С. В. Функции алгебры логики и классы Поста. М.: Наука, 1966.

[3] Захарова Е. Ю., Кудрявиев В. Б., Яблонский С. В. О предполных классах в k-значных логиках // ДАН. 1969. Т. 186. № 3. С. 509-512.

[4] Ивлев Ю. В. Модальная логика. М.: Изд-во Моск. ун-та, 1991.

[5] Карпенко A. С., Логики Лукасевича и простые числа, M.: URSS, 2007.

[6] Карпенко A. C. Многозначные логики / Сер. Логика и компьютер. М.: Наука, 1997.

[7] Раца M. Ф. О классе функций трехзначной логики, соответствующем первой матрице Яськовского. Кишинев. С. 185-213.

[8] Раца М. Ф. О классе функций логики, соответствующей первой матрице Яськовского // Исследования по общей алгебре. Кишинев. 1965. С. 99-110.

[9] Финн B. K. О критерии функциональной полноты в В3 // Исследования по формализованным языкам и неклассическим логикам. М.: Наука, 1974. С. 194199.

[10] Яблонский C. В. О функциональной полноте в трехзначном исчислении // ДАН. 1954. Т. 95. № 6. С. 1153-1155.

[11] Яблонский C. В. Введение в дискретную математику. М.: Наука, 1986.

[12] Яблонский C. В. Функциональные построения в k-значной логике // Труды математического института имени В. А. Стеклова. 1958. Т. 51. С. 5-142. 